\title{
Hexadecatetraenoic Acid n-1
}

National Cancer Institute

\section{Source}

National Cancer Institute. Hexadecatetraenoic Acid n-1. NCI Thesaurus. Code C68376.

A polyunsaturated long-chain fatty acid with a 16-carbon backbone and 4 double bonds,

originating from the 1st, 4th, 7th and 10th positions from the methyl end. 